\title{
INSAR TIME SERIES INVESTIGATION OF LAND SURFACE DEFORMATION IN AZAR OIL FIELD
}

\author{
Zahra Mirzaii ${ }^{1}$, Mahdi Hasanlou ${ }^{1 *}$, Sami Samieie-Esfahany ${ }^{1}$, Mahdi Rojhani ${ }^{2}$ and Parviz Ajourlou ${ }^{1}$ \\ ${ }^{1}$ School of Surveying and Geospatial Engineering, College of Engineering, University of Tehran; \\ 2 Department of Civil Engineering, Faculty of Engineering, Shahed University \\ (z_mirzaei, hasanlou, s.samieiesfahany, parviz.ajourlou)@ut.ac.ir, m.rojhani@shahed.ac.ir
}

\section{Commission VI, WG VI/4}

KEYWORDS: Persistent Scatterers Interferometry, Azar Oil Field, displacement, InSAR, oil extraction

\begin{abstract}
:
Time-series interferometric synthetic aperture radar (InSAR) has developed as an influential method to measure various surface deformations. One of the generations of time-series InSAR methodologies is Persistent Scatterer Interferometry (PSI) that focuses on targets with a high correlation over time. In this study, we have measured the surface deformation in Azar Oil Field utilizing time series analysis. Azar Oil Field is one of Iran's oil fields. This oil field is located in the east of the city of Mehran, Ilam province. The reservoir of this oil field is shared by Iraq oil field whose name is Badra where oil extraction started in 201409. While Iran started oil exploration in 201709, Iraq has maximized its oil exploration ever since. The subsidence is mainly observed in the vicinity of the oil field. The Stanford Method for Persistent Scatterers (StaMPS) package has been employed to process 20 descending ENVISAT-ASAR images collected between 2003 and 2009, as well as 50 descending Sentinel-1A satellite images collected between 2014 and 2019. Sentinel-1 images bring new improvements due to their wide coverage and high revisiting time, which allows us to make a wide area processing. Due to the high depth of oil wells (4,300 meters), as well as the stone type of the region's bed in some areas, we needed to calculate the magnitude of subsidence. The results show the maximum displacement rate in this area is $18 \mathrm{~mm}$ between 2014 and 2019 in the radar line of sight direction, but no subsidence took place between 2003 and 2009 .The results of the study confirm typical patterns of subsidence induced by oil extraction. Also, since 2017, with the onset of Iran's oil extraction and the intensification of Iraq's oil exploration, subsidence has taken place with a steeper slope. The displacement of the area before and after this date is modelled with two lines.
\end{abstract}

\section{INTRODUCTION}

Satellite radar Interferometry (InSAR) method has proven to be an effective remote sensing tool to map ground deformations from various natural causes or human activities such as volcano dynamics (Q. Luo et al. 2014), earthquakes (J. Yen et al. 2008), slope instability (L. Jiang et al. 2011), groundwater overexploitation (B. Yu et al. 2013), mining (H. Jung et al. 2007), and coastland reclamation (L. Jiang and H. Lin, 2010).

Any factor that can affect the phase of the backscattered radar signal can affect the deformation pattern. These measurements include surface displacements, land topography, land changes, land subsidence/uplift, water levels, soil moisture, snow accumulation, the volume of the forest, etc. Therefore, InSAR has found very broad applications in the field of earth and environmental sciences (X. Zhou et al. 2009).

Land surface deformation in Azar Oil Field has occurred due to various causes, including oil extraction, severe rains after a drought period, and the weak soil of the region.

Between 2007 and 2011, the region witnessed drastic falls in precipitation, but sharp increases from 2011 to 2013. The region's soil, considered to be of a weak type, is classed as sandstone in the international categorization. Since 2013, some cracks have formed which despite modifications still persist. Because of these observable changes, a lot of field information, such as geotechnical, hydrological, meteorological, seismic, and geophysical data, was collected in the area. In Figure 1. Azar Oil Field, 15 wells that were excavated, and the cracks formed in the region are shown.

Drilling from Iraq's petroleum tank started in 2014, and Iran began to drill in 2017. The oil in this tank is worth around four billion, and it is planned to drill the whole amount within a 20year period.

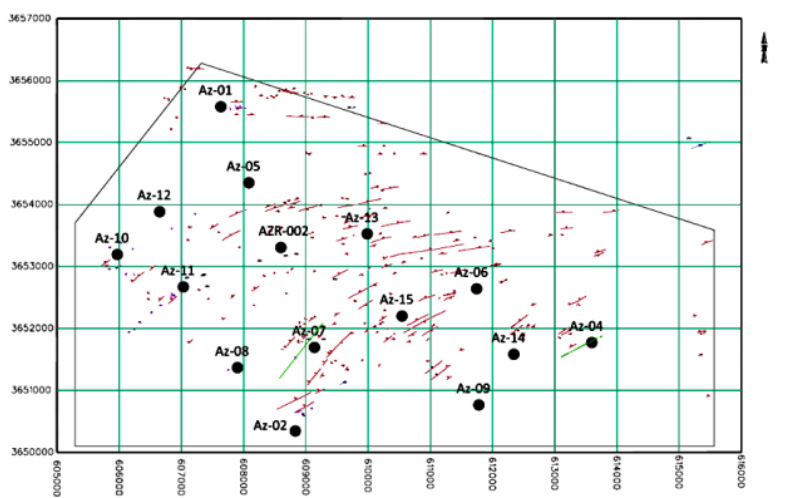

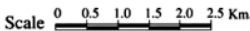

Figure 1. Distribution of wells and cracks in Azar oil field

The length of this tank is $36 \mathrm{~km}$ in the northeast, southwest direction, $13.5 \mathrm{~km}$ of which is located in Iran, and it extends along a width of $30 \mathrm{~km}$. Some cracks are shown in Figure 2.
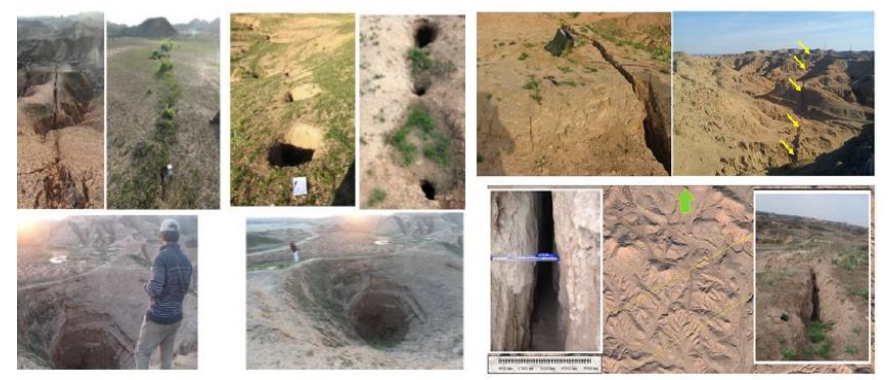

Figure 2. Sample of cracks observed in Azar Oil Field 
The resulting land surface deformation leads to widespread damage to oil and drilling rigs, roads and infrastructures, and significant economic loss.

Knowledge of the spatial and temporal extent of land settlement provides important information to establish effective measures necessary to mitigate losses from ground subsidence hazard (Motagh et al., 2008).

Our objective was to derive the temporal pattern of land deformation in this region using additional SAR data. C-band data from Copernicus missions Sentinel-1A have some attractive properties such as large volumes of archived imagery, larger coverage/swath, and importantly, from the application point of view, free and open access data policy. We utilize 50 descending Sentinel-1A satellite images collected between 2014 and 2019, as well as ENVISAT-ASAR images collected between 2003 and 2009. ENVISAR-ASAR was processed to determine whether or not any subsidence existed before these changes occurred.

\section{METHOD}

In a SAR image, every pixel is associated with a rectangular resolution cell on the ground. The data are sampled in an azimuth (i.e. flight direction) and a slant-range coordinate system, so every pixel has a unique range and an azimuth coordinate. Focused SAR images are stored in a standard format known as single-look complex (SLC) data (Samiei Esfahany, 2017). The idea of InSAR is based on the interference of two SAR images, acquired from different positions or at different times, exploiting mainly the phase difference between the two acquisitions. The phase difference between two images can be exploited to get information about the position of the target, or about the displacement during the time between two acquisitions. Interfering two SAR images is another complex image called an interferogram. before computing interferograms, it is required to align or co-register one image to the geometry of the other.

The interferometric phase $\phi_{M S}$ for a single pixel can be written as the summation of four components:

$\phi_{M S}=\mathrm{W}\left\{\psi_{M}-\psi_{S}\right\}=W\left\{\phi^{\text {range }}+\phi^{\text {atmo }}+\phi^{\text {scat }}+\phi^{\text {noise }}\right\}$

$M$ and $S$ indices stand for master and slave image that are two image complex conjugate together to make interferogram respectively where $\mathrm{W}\{$.$\} is the modulo- 2 \pi$ wrapping operator, $\phi^{\text {range }}$ the range-dependent phase related to the distance between the radar sensor and the effective phase center of the resolution cell on the ground, $\phi^{\text {atmo }}$ the phase delay induced by the atmosphere, $\phi^{\text {scat }}$ the scattering phase that is related to the distribution of all scatterers within a resolution cell, and $\phi^{\text {noise }}$ the additional system or thermal noise which is dependent on sensor specifications (Samiei Esfahany, 2017).

The phase component range, atmosphere scatter and noise can be further decomposed into some components that are shown in Figure 3.

In order to produce an interferogram from two SLC images, different processing steps are applied. Although there are various algorithms and processors available for interferometric processing, and they differ in some details and implementations, our InSAR process includes some key steps that include the following: (1) Co-registration and resampling, (2) Oversampling, (3) Flat earth and topographic phase subtraction (Samiei Esfahany, 2017). After generating interferograms with a single master, time series analysis requires us to find the persistent scatter to take the unwrapping step to remove phase ambiguity. The persistent scatter has some features that are stable over a long time and remain coherent over a sequence of the interferogram.

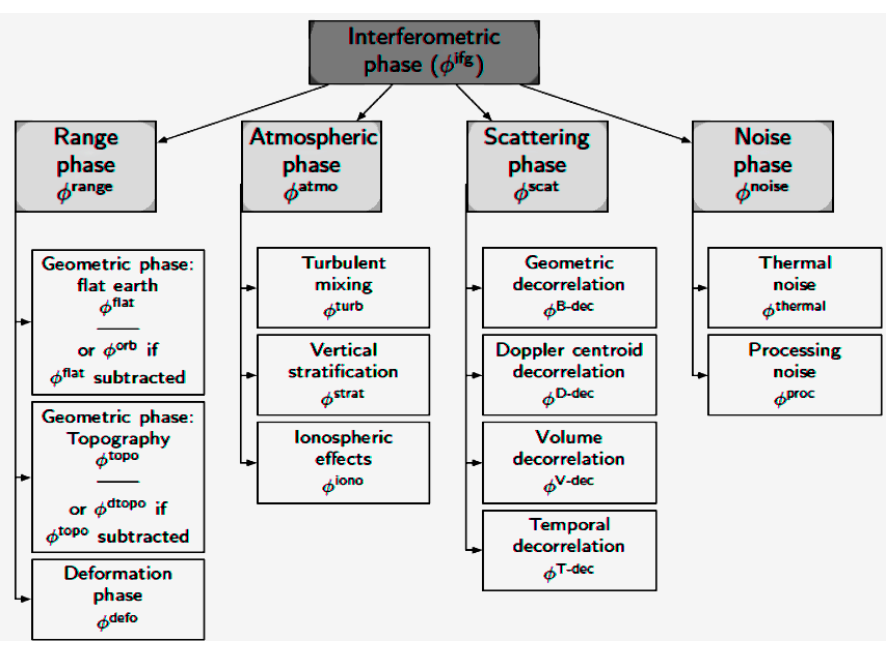

Figure 3. Decomposition of interferometric phase (Samiei Esfahany, 2017)

Although the details of these algorithms depend on the implementation, all of them contain three main processing blocks: coherent pixel selection, Spatio-temporal (3D) unwrapping, and atmospheric-signal mitigation (Ferretti et al. 2000; Kampes 2005). We demonstrate the work-flows of STAMPS PSI methods in Figure 4:

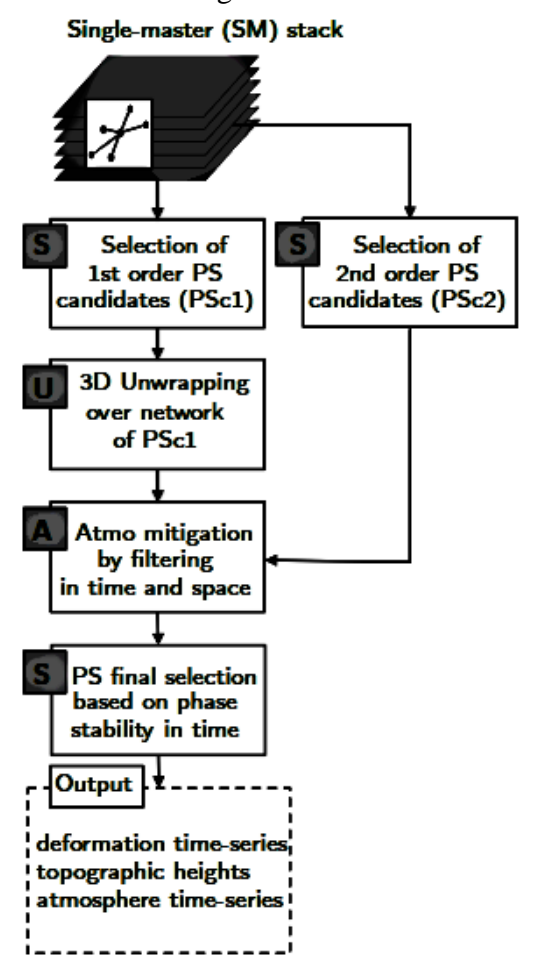

Figure 4. Generic flowchart of PSI processing. The steps are classified into Three basic InSAR processing blocks (expressed in the gray boxes) as S: pixel selection, U: unwrapping, A: atmospheric-signal mitigation

In this method, we used the phase stability with respect to a predefined model in space to select PS-pixels. After this selection of PS, 3D unwrapping was performed, and mitigation of atmospheric signal with high-pass filtering in time and low-pass filtering in space was performed.

Radar images of the study area were acquired by the European Space Agency's Sentinel1 and ENVISAT-ASAR. The dataset 
includes 20 images from the descending track 6 spanning the period between August 2003 and October 2010, 50 images from the descending track 6 covering October 2014-Feb 2019 that shown in Figure 5.

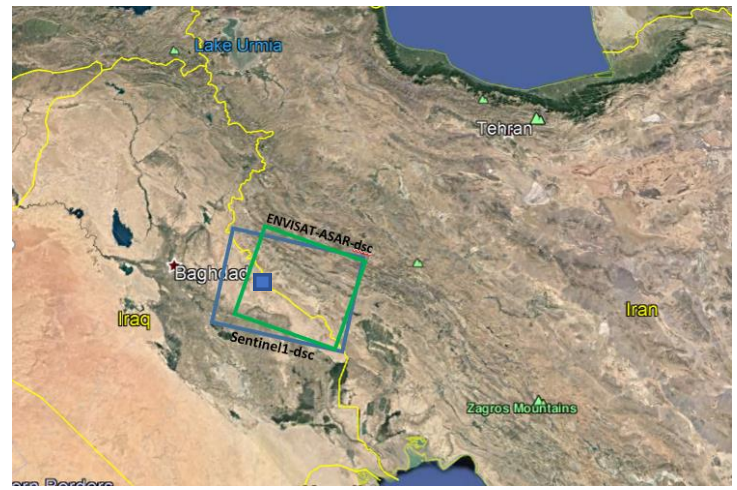

Figure 5. Topography map of Mehran, Ilam. The boxes show satellite trajectories of radar images for descending orbits. The blue box is Azar Oil Field

We processed the data in a single master with PSI. Interferometric combinations of the images were carried out with the consideration of their spatial and temporal baselines. The overview of the baseline perpendicular plot of the images can be seen in Figure 6.

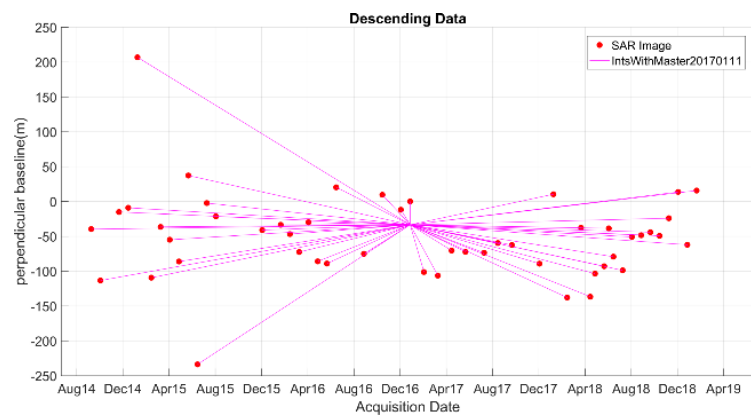

(a)

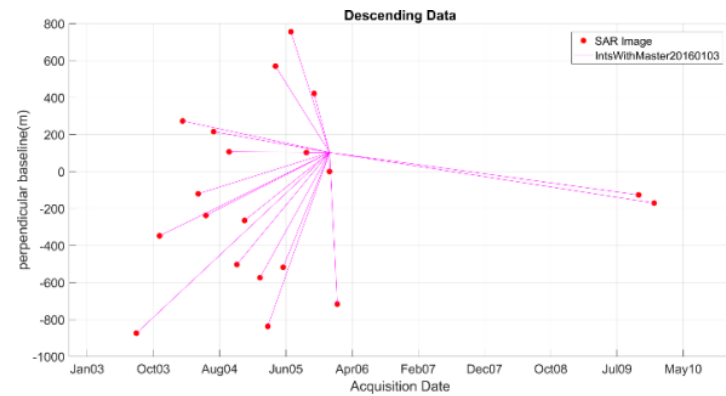

(b)

Figure 6. Plots of acquisition dates versus perpendicular baselines for the final network of the (a) S1 descending, (b) ENVISAT descending, data used for the time-series analysis. The circles represent SAR images, and the lines represent the single master interferograms.

The interferograms were corrected for the phase signature due to orbital separation using precise orbits. To eliminate the topographic component from the differential interferograms, we used an external DEM generated from NASA's SRTM. The resulting interferograms were used to derive the time series of the deformation field. Pixel selection, unwrapping, and atmosphericsignal mitigation were carried out with Stanford Method for Persistent Scatterers (StaMPS) package.

\section{RESULTS}

Deformation velocity maps in Iran-Iraq Joint oil field and Azar Oil Field are shown in Figure 7.

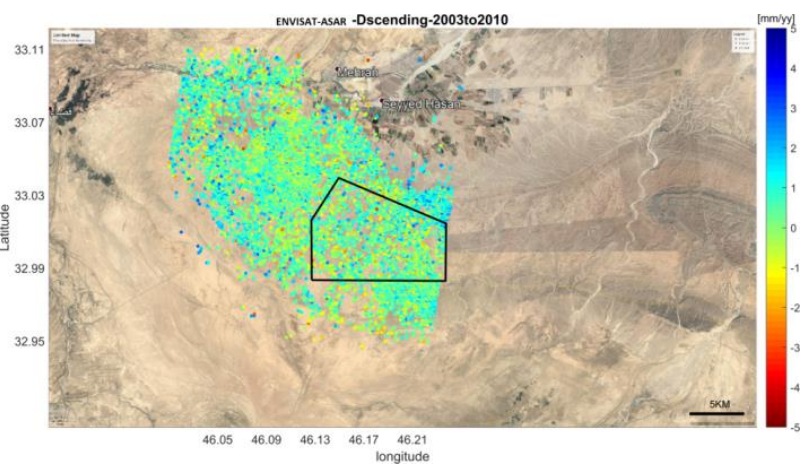

(a)

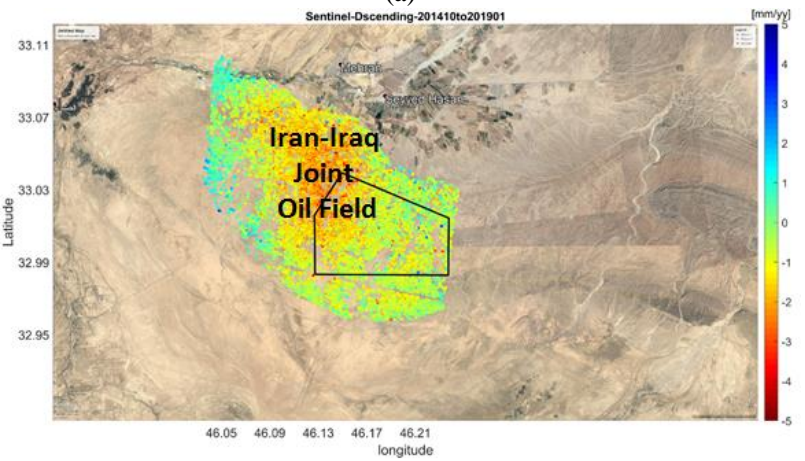

(b)

Figure 7. Deformation velocity map (a) ENVISAT-ASAR between 2003 to 2009 with reference point $(46.22,33.02)$ (b) Sentinel-1 between 2014 to 2019 with reference point (46.22, 33.02)

As you can see in Figure 7, no displacement is observed between 2003 and 2009, but from 2014 to 2019, owing to three years of drought and subsequent precipitations, as well as the region's soil type and oil drilling, a 4.5-mm displacement per year, can be seen. The cumulative displacement diagram (Figure 8) for the four-year period is presented below.

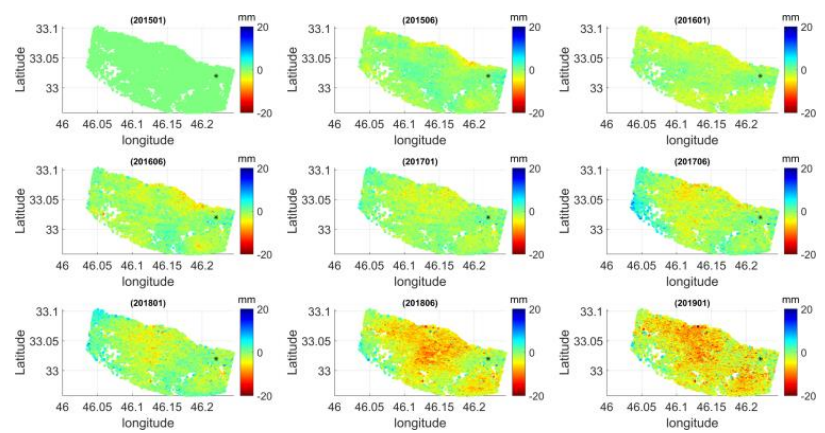

Figure 8. Displacement time-series for Azar area obtained from the PS analysis of the descending Sentinel-1 
We obtained $\sim 18 \mathrm{~cm}$ subsidence during 4 years in this tank. The displacement trend for 100 points was analyzed and is shown in Figure 9 in the Results section.

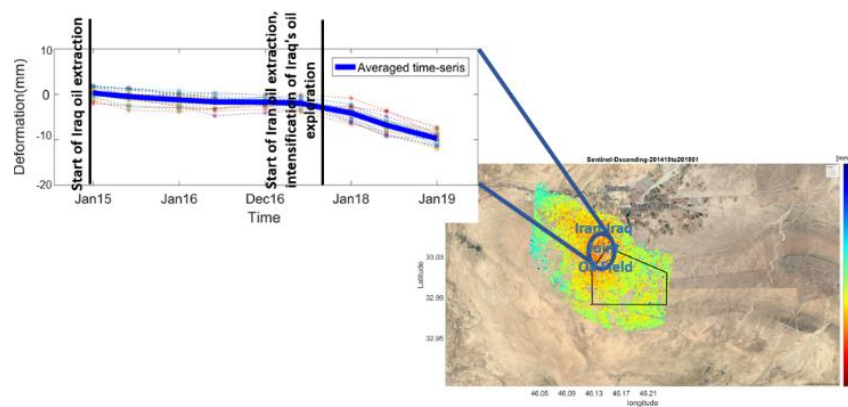

Figure 9. displacement trend for 100 points over time

The results, obtained by processing radar images through PSI methods, indicate a 4.5-mm subsidence per year in the line of sight direction. The region's subsidence is due to oil drilling, considerable precipitations, and the weak soil type. Oil drilling is of paramount importance, and serious measures need to be taken to inspect and control this subsidence. By using specimen from 100 points in the region shown in Figure 9, we modeled this land deformation in Figure 10. R2 of the first line is 0.8948 and for the second line 0.9954 . These correlation coefficients represent the good fitting of the line and the accuracy of the fitted model.

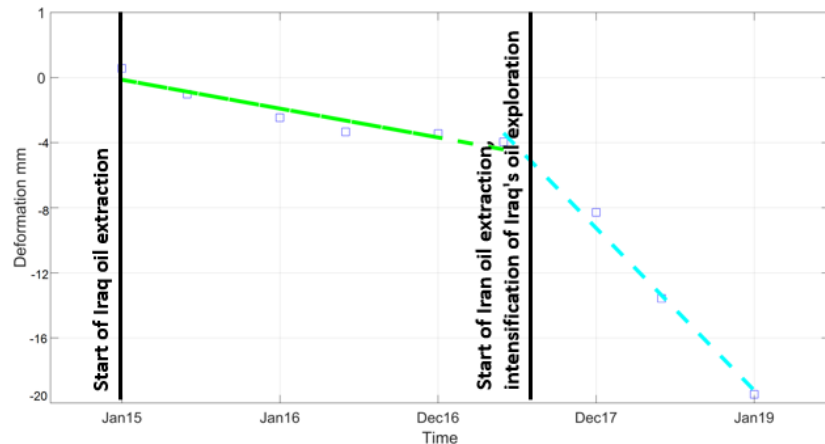

Figure 10. Line fitting or modeling for displacement time-series in Azar area

\section{CONCLUSION}

This study presents an application of the InSAR time series analysis to derive the spatial-temporal evolution of land subsidence in Azar Oil Field. The PSI method was applied for a dataset of 49 interferograms in order to extract surface deformations. The analyses show that oil extraction plays a key role in land subsidence in Azar Oil Field. Also, since 2017, with the onset of Iran's oil extraction and the intensification of Iraq's oil exploration, subsidence has taken place with a steeper slope. Due to the high depth of oil wells (4,300 meters), we expect that subsidence covers the large area and has a small magnitude. The results of InSAR time-series analysis confirm patterns of subsidence induced by oil extraction.

\section{REFERENCES}

Q. Luo, D. Perissin, H. Lin, Y. Zhang and W. Wang, 2014. Subsidence Monitoring of Tianjin Suburbs by TerraSAR-X Persistent Scatterers Interferometry, IEEE Journal of Selected Topics in Applied Earth Observations and Remote Sensing, vol. 7, no. 5, pp. 1642-1650.
J. Yen, K. Chen, C. Chang and W. Boerner, 2008. Evaluation of earthquake potential and surface deformation by Differential Interferometry, Remote Sensing of Environment, vol. 112, no. 3, pp. 782-795.

L. Jiang, H. Lin, J. Ma, B. Kong and Y. Wang, 2011. Potential of small-baseline SAR interferometry for monitoring land subsidence related to underground coal fires: Wuda (Northern China) case study, Remote Sensing of Environment, vol. 115, no. 2, pp. 257-268.

B. Yu, G. Liu, Z. Li, R. Zhang, H. Jia, X. Wang and G. Cai, 2013. Subsidence detection by TerraSAR-X interferometry on a network of natural persistent scatterers and artificial corner reflectors, Computers \& Geosciences, vol. 58, pp. 126-136.

H. Jung, S. Kim, H. Jung, K. Min and J. Won, 2007. Satellite observation of coal mining subsidence by persistent scatterer analysis, Engineering Geology, vol. 92, no. 1-2, pp. 1-13.

L. Jiang and H. Lin, 2010. Integrated analysis of SAR interferometric and geological data for investigating long-term reclamation settlement of Chek Lap Kok Airport, Hong Kong, Engineering Geology, vol. 110, no. 3-4, pp. 77-92.

M. Motagh, et al., 2008. "Land subsidence in Iran caused by widespread water reservoir overexploitation," J. Geophys. Res letters, vol. 35, L16403, doi:10.1029/2008GL033814.

Sami Samiei Esfahany, (2017). Exploitation of Distributed Scatterers in Synthetic Aperture Radar Interferometry. PhD thesis, Delft University of Technology, Delft, the Netherlands.

Ferretti, A., Prati, C., and Rocca, F. (2000). Nonlinear subsidence rate estimation using permanent scatterers in differential SAR interferometry. IEEE Transactions on Geoscience and Remote Sensing, 38(5):2202-2212.

Kampes, B. (2005). Displacement Parameter Estimation using Permanent Scatterer Interferometry. PhD thesis, Delft University of Technology, Delft, the Netherlands.

X. Zhou, N. Chang and S. Li, 2009. Applications of SAR Interferometry in Earth and Environmental Science Research, Sensors, vol. 9, no. 3, pp. 1876-1912. 